\title{
MORPHOLOGY OF CORPORA LUTEA IN THE UGANDA KOB ANTELOPE, ADENOTA KOB THOMASI (NEUMANN)
}

\author{
J. A. MORRISON \\ Oklahoma Cooperative Wildlife Research Unit, \\ Oklahoma State University, Stillwater, Oklahoma, U.S.A.
}

(Received 27th Fuly 1970, revised 26th February 1971)

Summary. Observations on ninety-five individually marked female Uganda kob revealed that oestrus may recur one or more times at 6to 13-day intervals. Necropsies of 197 pregnant and 144 non-pregnant females disclosed that many pregnant females were lactating, indicating that post partum anoestrous intervals are brief, and ovaries from a nonpregnant female may contain abundant CL existing concurrently in apparently different stages of development and regression. To aid in interpreting these phenomena, CL from all stages of reproduction were studied microscopically.

Pregnancy CL are typical of artiodactylan corpora in general. Their size changes little during pregnancy, and ranges from 10 to $13.8 \mathrm{~mm}$ in diameter. Luteal cells commence regression after the 31 st week of the 35week gestation period; CL of pregnancy degenerate rapidly in histology after parturition, measuring 6 to $11 \mathrm{~mm}$ in diameter until 10 days post partum, then decrease markedly to $<5 \mathrm{~mm}$ within the next 30 days.

Cyclic CL in non-pregnant females are 2.5 to $9 \mathrm{~mm}$ in diameter. Distinct ovulation stigmata are present on more than two-thirds of these GL. Two or more immature corpora of different ages may exist concurrently with several regressive $\mathrm{CL}$ in a non-pregnant female. Over half of these begin regressing while histologically immature and incompletely developed.

\section{INTRODUCTION}

In an earlier study, Buechner (1961a, 1963) found that Uganda kob antelope, Adenota kob thomasi (Neumann), on the Semliki Plains of western Uganda, exhibit arena-like mating behaviour in which the oestrous female leaves the herd and goes to a territorial mating ground to copulate with a male holding one of the thirty to forty individual territories comprising the mating ground. Mating is restricted to the territorial mating ground and is non-seasonal. Monotocous births occur at a uniform rate in the population throughout the year. These disclosures led to another investigation to study territorial mating as an adaptation of the population to its environment. In these studies, characteristics of reproduction were found that are not commonly attributed to wild artiodactyls: (1) many lactating females, often nursing extremely young fawns, 
were pregnant or in oestrus, suggesting that post partum anoestrous intervals are brief; (2) marked females of known breeding histories were in oestrus two or more consecutive times which, in several instances, were at intervals of 6 to 13 days; and (3) non-pregnant females in general contained unusually abundant CL existing concurrently in various combinations of what appeared macroscopically to be recently ovulated follicles, newly developing CL, matureappearing CL which were significantly smaller than the CL of pregnancy, and a greater quantity of regressed CL than could have been retained from the previous pregnancy.

These phenomena suggest that although kob may have a short post partum anoestrous interval, conception is preceded by repetitions of infertile oestrus and ovulation. Furthermore, CL in different stages of development probably exist concurrently because they originate in the oestrous periods repeated at 6- to 13-day intervals. The significance of these phenomena is that large artiodactyls are said, in the literature, to have lengthy anoestrous intervals while lactating and to have dioestrous intervals of approximately 21 days. The CL formed in a given ovulation grow to full size and histological maturity, then regress markedly before the next ovulation takes place and a new cL appears.

The kob apparently differs markedly from this pattern. The phenomena observed in kob were analysed by correlating ovarian morphology with behavioural events occurring in the reproductive cycle. This was done by histological study and is the subject of the present report. The results shown here are employed in another article to illustrate the post partum anoestrous interval and the repetitions of oestrus in question (Morrison \& Buechner, 1971).

\section{MATERIALS AND METHODS}

Complete reproductive tracts were taken from 197 pregnant and 144 nonpregnant females collected during 1957 to 1958 and 1962 to 1964. The kob were culled during a meat-cropping programme of the Uganda Game Department and by special permission of the Uganda Government. Reproductive activity was described by observing the mating behaviour of ninety-five individually marked females from towers erected near three territorial mating grounds. Such females were later found for collection by searching through the feeding herds near the mating ground. While observing the feeding herds, fifty-one new fawns were caught and marked within 1 to 3 days post partum. Eleven of these fawns were subsequently found again to collect their mothers at known post partum intervals.

Immediately after the kob were shot, their body measurements were taken and all tissues kept for study were fixed in $10 \%$ formalin. Concepti were dissected into major components for measurement and preservation.

Ovaries collected in 1957 and 1958 were embedded in a medium of gelatin and corn meal (Pimlott \& Mossman, 1959); the ovaries and the enveloping medium were then sliced into 2-mm sections for macroscopic examination. Ovaries collected in 1962 to 1964 were not embedded, leaving ovulation sites uncovered for study, but they were sliced into 2-mm sections. The size of each CL was measured as an average of its length, width and height. Paraffin wax- 
embedded samples of cL were sectioned at $7 \mu \mathrm{m}$ and stained with Groat's tetrachrome for histological study. Corpora lutea of pregnancy are included to serve as the standard of mature CL in comparison to cyclic GL of ovulation in non-pregnant females.

The histological characteristics employed here to evaluate growth or regression in CL were adapted from studies on the ewe (Marshall, 1903; Warbritton, 1934), sow (Corner, 1919, 1921), cow (McNutt, 1924; Hammond, 1927; Moss, Wrenn \& Sykes, 1954) and goat (Harrison, 1948). Typical changes in the morphology and size of luteal cells accompany development or regression in $\mathrm{CL}$, and luteal cells in the kob were classified proportionally according to five types of development adapted to kob from criteria described in cattle by Foley $\&$ Greenstein (1958); average cell and nucleus dimensions $(\mu \mathrm{m} \pm$ S.E.) given here are of kob luteal cells.

\section{Type I}

Cells are partly developed; angular, stellate or cylindrical. Their walls or outlines are indistinct and the cells are not closely apposed. The cytoplasm stains very light orange, is finely granular, homogeneous and rarely pigmented or vacuolated. Nuclei are central, round or oval and vesicular and contain one or more 2- to 3- $\mu \mathrm{m}$ nucleoli. Karyolymph is clear and chromatin is finely granular and dispersed to the nuclear membrane. Cell dimensions are $26.2 \pm 1.65$ $\times 13.5 \pm 0 \cdot 79$; nuclear dimensions are $10 \cdot 4 \pm 0 \cdot 14 \times 8 \cdot 9 \pm 0.53$.

\section{Type II}

Cells are fully mature, oval or round, have distinct walls and are closely apposed. The cytoplasm is dense, homogeneous and granular, occasionally pigmented or slightly vacuolated and stains pale orange. Nuclei are central, round or oval, vesicular and contain one or more nucleoli. Karyolymph is clear and chromatin is finely granular and dispersed to the nuclear membrane. Cells: $33 \cdot 8 \pm 0 \cdot 60 \times 24 \cdot 5 \pm 0 \cdot 42$; nuclei: $9 \cdot 7 \pm 0 \cdot 11 \times 8 \cdot 6 \pm 0 \cdot 11$.

\section{Type III}

Cells have commenced regressing and have irregular shapes. Their walls are contracted, wrinkled and disintegrated in places. The intercellular spaces are widening. The cytoplasm is foamy with profuse small vacuoles or may have few large $(5 \mu \mathrm{m})$ vacuoles. It stains dark orange and pigment crystals or droplets are abundant. The cytoplasmic density and staining quality are variable. Nuclei may be full size but with denser membrane or may be crenated, contracted, and with coarse chromatin. Cells: $29 \cdot 7 \pm 0 \cdot 87 \times 23 \cdot 1 \pm 0 \cdot 67$; nuclei: $7 \cdot 6 \pm 0 \cdot 11 \times 6 \cdot 4 \pm 0 \cdot 15$.

\section{Type IV}

Cell degeneration is advanced. Cell size and shape are irregular, mostly stellate with contracted, wrinkled, partly disintegrated walls. The cells are widely separated. The cytoplasm stains darkly, is reduced to strands or nets around abundant vacuoles and pigments are abundant. Nuclei are dark, pycnotic, irregularly shaped, eccentrically positioned in the cell and may occupy 
much of contracted cell body. Chromatin is coarse and condensed. Cells: $25 \cdot 8 \pm 1 \cdot 23 \times 18 \cdot 3 \pm 0 \cdot 61$; nuclei: $7 \cdot 1 \pm 0 \cdot 16 \times 5 \cdot 8 \pm 0 \cdot 22$.

\section{Type $V$}

Degeneration is highly advanced. Cell size and shape are extremely irregular. Cell walls are absent or only present as wrinkled fragments. Cytoplasm is compacted into a golden-orange mass of pigment. Nuclei are much contracted, hyperchromatic, have coarse chromatin, or may be disintegrated. Cells: $15 \cdot 8 \pm 0 \cdot 77 \times 9 \cdot 7 \pm 0 \cdot 43$; nuclei: $8 \cdot 7 \pm 0 \cdot 34 \times 5 \cdot 6 \pm 0 \cdot 20$.

\section{RESULTS}

\section{Cyclic corpora lutea of ovulation}

The macroscopic characteristics of these cL suggested that they belonged to several classes or stages of formation, growth and regression. The validity of the classes was tested by histological examination. The classes are designated by letter for convenience of reference and are presented in order of development.

Class $A$. These cL included recently ovulated follicles at the beginning of formation. They were $5.9 \pm 0.33 \mathrm{~mm}$ in diameter (about half as large as mature follicles) but retained patent antra into which infoldings of the convoluted follicle wall extended as white (after fixation in formalin) trabeculae (Pl. 1, Fig. 1). The trabeculae may extend to the cL centre and, although separate, loosely fill the antrum. Large rupture sites were present on all cL. The membrana granulosa contained undifferentiated granulosa cells of $7 \times 10 \mu \mathrm{m}$ (Pl. 1, Fig. 2). Extravasated erythrocytes were abundant in the antrum. Leucocytes were numerous in the theca interna, theca externa and trabeculae. A few, short, unbranched capillaries and connective tissue fibrils entered the membrana granulosa from the theca interna.

Class $B$. The trabeculae were expanded to fill the antrum completely and were united into a white mass. The CL dimensions averaged $6 \cdot 6 \pm 0.34 \mathrm{~mm}$, and the CL were spherical or oval, and lay at the ovarian periphery. Ovulation stigmata were identifiable on $67 \%$ of the CL. Ingrowths of the theca interna which had penetrated into the trabeculae remained as septa radiating to the CL centre between segments of luteal tissue. A solid mass of connective tissue or a 2- to 3-mm cavity lined with connective tissue occupied the cL centre and connected with the septa. The thecae were convoluted in various degrees. Extravasated erythrocytes and leucocytes were less common than in Class A. Arterioles and venules extended into the septa, and capillaries lay between parallel rows or clumps of luteal cells.

In half of the sample of Class-B cL, the luteal tissue (Pl. I, Fig. 3) consisted of undifferentiated granulosa cells at the cL centre and Type I cells at the periphery. Many cells lay in parallel rows radiating inward from the septa or theca interna, or were arranged in large clumps. Numerous connective tissue fibrils extended linearly between luteal cell rows or clumps. In the other half of the sample, the CL contained evidence of regression (PI. 1, Fig. 4). Their luteal cells were approximately $50 \%$ of Type I, $25 \%$ of Type II, and $25 \%$ of Type III. The centre of the $\mathrm{GL}$ might contain undifferentiated granulosa cells, and Type 
PLATE 1
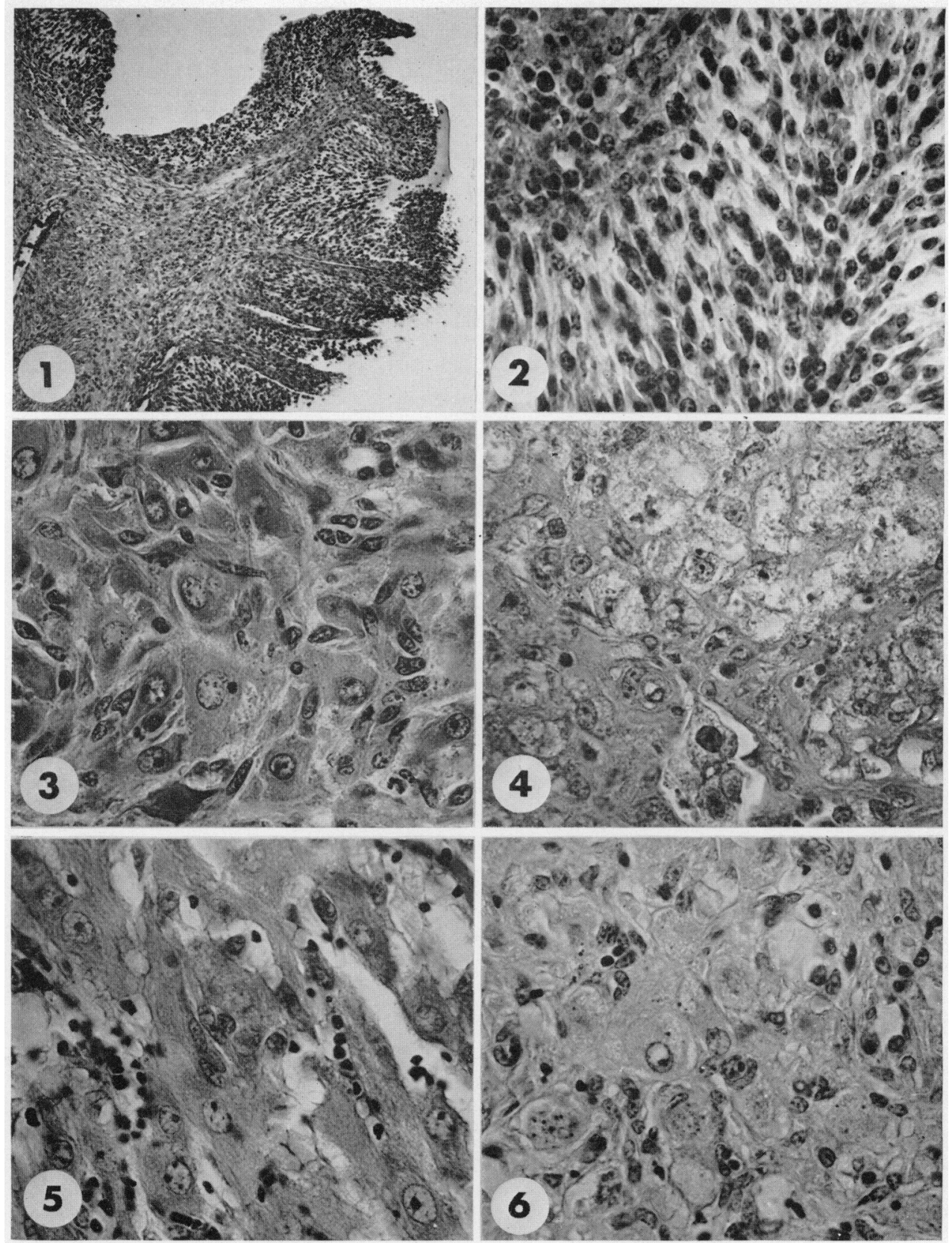

FIs. 1. Corpus luteum, Class $\Lambda$. Newly forming trabeculum projecting into antrum. $\times 60$.

Fic. 2. Corpus luteum, Class $A$. Cells of the membrana granulosum commencing growth. $\times 400$.

Fic. 3. Corpus luteum, Class B. Non-regressive, lutcinization progressing. $\times 400$.

FIG. 4. Corpus lutcum, Class B. Regressive. $\times 400$.

Fif, 5, Corpus luteum, Class $C$. Non-regressive. $\times 400$.

Fic. 6. Corpus luteum, Class C. Regressive. $\times 400$.

(Facing p. 300) 

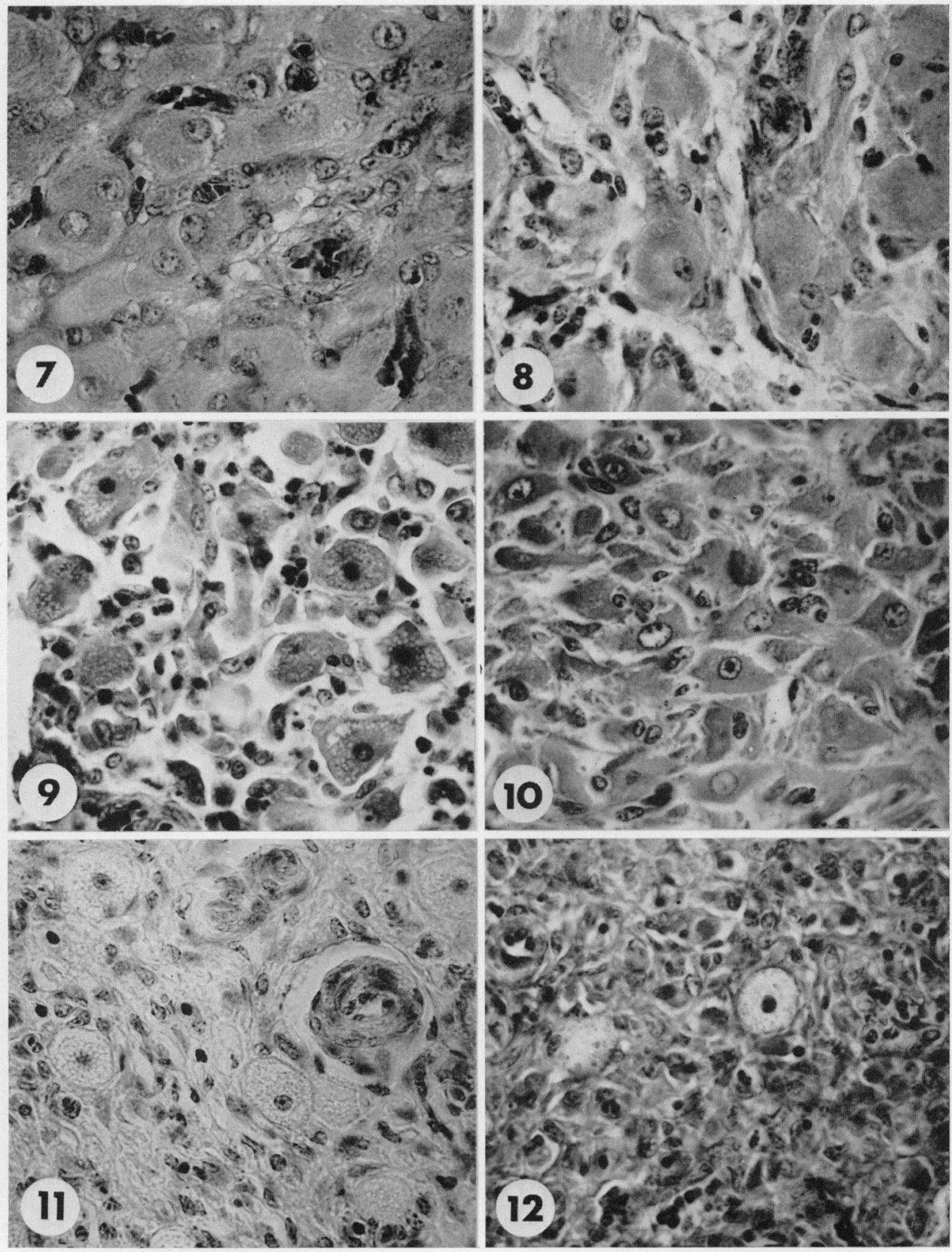

Fig. 7. Corpus luteum of pregnancy, approximately 15 to 20 days post coitum. $\times 400$.

Fig. 8. Corpus luteum of pregnancy, 216 days gestation. $\times 400$.

Fis. 9. Corpus luteum of pregnancy, 1 day prost frattum. $\times 400$.

Fic. 10. Corpus luteum of pregnancy, 5 to 10 days prost partum. $\times 400$.

Fig. 11. Corpus luteum, Class D. $\times 400$.

FIG. 12. Corpus lutcum, Class E. $\times 400$. 
IV cells might lie at the periphery. The walls of larger vessels and interstitial connective tissue fibrils were hypertrophied and hyaline tissue had formed interstitially.

Class $C$. These cL were yellow, spherical or oval, peripherally situated in the ovary, averaged $5 \cdot 8 \pm 0.30 \mathrm{~mm}$, and $68 \%$ had visible ovulation stigmata. Except for their small size, they were macroscopically similar to cL of pregnancy. The thecae were distended from growth of luteal tissue and were well vascularized. The septa were prominent and divided the luteal tissue into segments.

In $40 \%$ of the cL, the luteal cells were mainly Type II with some Type I cells at the centre (Pl. 1, Fig. 5). Many CL had areas in which the luteal cells were arranged in linear, parallel rows. Leucocytes were absent. Capillaries extended linearly, seldom branching, between rows and clumps of luteal cells. Larger blood vessels ( $>50 \mu \mathrm{m}$ in diameter) were restricted to the thecae and septa. Connective tissue fibrils ramified around clumps of luteal cells. The cL centre might contain a connective tissue mass or lined cavity, joined by the septa, or contained luteal tissue only.

In the remaining $60 \%$ of the sample, $25 \%$ of the cells in the luteal tissue were partly undifferentiated granulosa cells or developing Type I cells, $50 \%$ were Type I, extremely few were of Type II, and the remainder were Types III and IV (Pl. 1, Fig. 6). Leucocytes occurred in the theca interna and septa; the thecae were interrupted by degenerating segments. Capillaries were disintegrated, leaving extravasated erythrocytes in situ; the large vessels had hypertrophied walls. The interstitial connective tissue fibrils were hypertrophied and coarse; dense bands of hyaline tissue lay between the luteal cells. These cL had regressed considerably.

Class $D$. All cL were bright, pale orange, spherical or oval, $3.5 \pm 0.17 \mathrm{~mm}$, and $62 \%$ had ovulation stigmata. A cL might have a peripheral connective tissue capsule $<1 \mathrm{~mm}$ thick, or contain several intracorporeal masses of connective tissue up to $2 \mathrm{~mm}$ in diameter. The most prevalent luteal cells were Type III; Types IV and V were common (Pl. 2, Fig. 11). Leucocytes were absent or extremely rare. The thecae were wrinkled and broken. Septa were absent, reticular connective tissue fibrils were extremely hypertrophied and dissociated and fibroblasts were abundant. Capillaries were extensively disintegrated and many short rows of erythrocytes were exposed in situ.

Class $E$. The colour of these cells was dark, dull, orange, and their size averaged $2.9 \pm 0.21 \mathrm{~mm}$. They were spherical, oval or flat, and visible ovulation stigmata were identifiable on $71 \%$ of the sample. Luteal cells were Type V which were either widely separated or compressed and overlapping (Pl. 2, Fig. 12). Leucocytes were absent. Thecae were largely disintegrated. Vascular regression was more extensive than in Class D. Fibroblasts were extremely abundant, filling the interstitial spaces, and reticular fibres were disintegrated in large areas.

Class $F$. All cells were $<2.0 \mathrm{~mm}$, dark brown, mainly cuboidal or flattened and disc-like. Their position in the ovary was variable. Ovulation stigmata were obliterated or dissociated from the corpora. Luteal cells and the thecae were completely disintegrated. These were corpora albicantia composed of 
highly compacted masses of connective tissue, blood vessels and luteal cell pigments.

\section{Corpora lutea of pregnancy}

Corpora lutea of pregnancy in kob are similar to artiodactylan CL in general. They occur singly, kob being monotocous, and are distributed equally between right and left ovaries (Buechner, 1961b). The majority of CL were oval, some were spherical, others were pear-shaped and occasionally positioned with the larger end toward the ovarian cortex. They were light to dark yellow after fixation in formalin. Three-quarters of the CL protruded from one- to twothirds of their diameters outside the ovarian cortex with the protruding portion covered by the tunica albuginea. The positions of non-protruding CL were variable, ranging from peripheral to central. Shades of colour, shapes, positions, and amounts of protrusion were independent of the stage of gestation.

Ante partum $C L$ of pregnancy (embryos microscopic). Each of twenty-one lactating females lacking a macroscopic embryo had a single, full-size, apparently mature cL. Ovaries from nineteen of these females were available for study. Five were collected at known intervals ranging from 25 to 70 days post partum. The CL from these ovaries averaged $11.2 \pm 0.22 \mathrm{~mm}$ in diameter and were macroscopically indistinguishable from known CL of pregnancy. As tested by Student's $t$ test, these cL did not differ $(P>0.05)$ in mean diameter from either CL of $<25$ days of known pregnancy $(11.4 \pm 0.16 \mathrm{~mm})$ or from cL of 25 to 50 days of pregnancy $(11 \cdot 7 \pm 0 \cdot 13 \mathrm{~mm})$. They were significantly smaller than cL of 195 to 245 days $(11 \cdot 8 \pm 0.08 \mathrm{~mm})$ and significantly larger than CL of pregnancy collected at 1 to 10 days post partum $(8.5 \pm 0.44 \mathrm{~mm})(P<0.05)$.

Luteal cells were mostly of Type II with some Type I cells located centrally in the cL. The cells were closely apposed, arranged in clumps, and many had indistinct cell outlines because cell walls were invisible or interstitial elements were undeveloped. The thecae were fully distended and markedly vascularized; septa were well-developed and contained large vessels. Few septa extended to the cL centre, and few CL retained central masses or cavities of connective tissue. Fifty per cent of the cL contained some leucocytes at the bases of the septa and in the theca interna. Mature vessels ( $>100 \mu \mathrm{m}$ in diameter) penetrated the luteal tissue; branched, reticulate capillaries were more abundant among luteal cells than in Class-C cL. Reticular connective tissue fibrils were also more extensive than in Class $\mathrm{C}$, but, as with capillaries, they were not as extensively developed between luteal cells as they were in older CL (PI. 2, Fig. 7).

Ante partum $C L$ of pregnancy (embryos macroscopic). The gestational stages of 168 pregnant females collected at random were estimated by ageing their foetuses with a growth curve based on thirteen foetuses of known age (Buechner, Morrison \& Leuthold, 1966). By chance, the sample was distributed evenly throughout the gestation period, which lasts for 235 to 240 days. Corpora lutea of pregnancy ranged in size from 10 to $13.8 \mathrm{~mm}$, with a mean diameter of $11.6 \pm 0.02 \mathrm{~mm}$. After embryos became macroscopic, CL size did not vary significantly according to a one-factor analysis of variance $(P>0.05)$.

Luteal cells at $<25$ days of gestation were predominantly Type II but included a few of Type I, and cell boundaries were less distinct than in older CL. After 25 
days, all cells were of Type II and had distinct outlines of endothelia, capillaries and fibrils. After 215 days of pregnancy, $75 \%$ of the luteal cells were vacuolated extensively but otherwise were Type II. The remainder were mainly Type III but included some Type IV. Near parturition (Pl. 2, Fig. 8), the cytoplasm of all luteal cells contained abundant pigment granules. Leucocytes were absent. The thecae were heavily vascularized. Septa were much reduced or absent, their places being taken by large blood vessels from which capillaries ramified around individual luteal cells. The interstitial reticulum of connective tissue fibrils also enclosed each cell.

Post partum $C L$ of pregnancy. Five non-pregnant females were collected at known post partum intervals of $1,20,25,27$ and 30 days. Ten others were collected at post partum intervals estimated to be 2 to 20 days by comparing the extent of their uterine regression with that in the five females of known intervals.

Corpora lutea of pregnancy collected at post partum intervals of 1 to 10 days were yellow and closely resembled the ante partum CL of pregnancy except for being smaller in size. Corpora lutea decreased gradually in diameter until about 10 days post partum, after which they shrank rapidly. Nine cL of pregnancy $<10$ days post partum ranged from 5.8 to $10.8 \mathrm{~mm}$ in diameter with a mean of $8.5 \pm 0.44 \mathrm{~mm}$. At intervals of 10 to 30 days post partum, CL were 3.0 to $4.8 \mathrm{~mm}$ with a mean of $4.2 \pm 0.27 \mathrm{~mm}$, and were orange or brown in colour.

The cL of pregnancy at $<10$ days post partum (Pl. 2, Figs. 9 and 10) largely contained Type III luteal cells but cells of Type IV were also found. The cells had shrunk, leaving wide interstitial spaces in which fibroblasts were numerous and reticular connective tissue fibres were enlarged and separated. The endothelium around the luteal cells had thickened. Capillaries were broken, extravasated leucocytes were numerous, and the walls of large blood vessels were hypertrophied. Corpora lutea of pregnancy at $>10$ days post partum could not be distinguished reliably from Class-D or Class-E GL by the methods employed here.

\section{DISCUSSION}

Before the corpora could be employed reliably to interpret specific phenomena in the reproductive biology of kob (Morrison \& Buechner, 1971), it was necessary first to resolve the identities of corpora in non-pregnant females and in females believed to be newly pregnant with microscopic embryos. A primary need was to establish that corpora were valid cL and not merely luteinized follicles. The high incidence of ovulation stigmata and the overall histological characteristics of corpora in non-pregnant females verified that they were true GL resulting from ovulation.

Although cyclic CL of ovulation do not enlarge to mature size, they occur in a variety of gross characteristics that suggest progressive stages of development and regression. Histological study confirmed the stages of development assumed from macroscopic appearance, showing that GL begin with the characteristics of Class A, are in immature but progressively older growth in Classes B and C, and are increasingly regressive through Classes $\mathrm{D}$ to $\mathrm{F}$. Microscopic examination also revealed that a large proportion of $\mathrm{CL}$ in Classes $\mathrm{B}$ and $\mathrm{C}$ are beginning 
to degenerate while yet immature, which undoubtedly accounts for their failure to reach full size. These aL closely resemble the degenerating, immature CL induced in cattle by Armstrong \& Hansel (1959), cL which were accompanied by shortened dioestrous intervals. The relationship of regressiveimmature ch to the 6- to 13-day dioestrous intervals in kob is discussed in another article (Morrison \& Buechner, 1971). It is sufficient here to emphasize that in female kob possessing cL of two or more different classes at the same time, the CL are derived from separate oestrous periods.

Pregnant females do not contain CL of Classes A, B or C although they do have CL of Class D or older. Regressive Class-C CL apparently regress into Class D by the time embryos are macroscopic; non-regressive Class-C CL either mature to full size or begin to regress at an age beyond that of Class- $\mathrm{C}$ cL in the collection.

Corpora lutea having the mature size and gross appearance of CL of pregnancy were present in thirty-four females lacking macroscopic embryos. Conditions in the field made it impractical to flush their uteri and uterine tubes to recover microscopic embryos, consequently their cL were compared histologically to each other and to known GL of pregnancy to ascertain which were full-size cyclic CL of ovulation, if any, newly formed CL of pregnancy, or CL of pregnancy collected shortly after parturition.

The mature-appearing cL in nineteen of these females are believed to represent new pregnancies rather than infertile ovulations because the CL are histologically uniform and extremely similar to GL of pregnancy known to be of $<25$ days gestation, and are unaccompanied by CL of Classes A, B or C. Full-sized CL of ovulation described in other artiodactyls commence regression shortly after reaching a peak of maturity and are strongly regressed by the end of the dioestrous interval. If these nineteen CL were from infertile ovulations, it is reasonable to expect that some of them would be regressive. The ages of these GL are probably about 14 days or less, because a female culled at 20 days post coitum contained a trophoblast $40 \mathrm{~mm}$ in length.

The importance of distinguishing CL of pregnancy accompanied by microscopic embryos from post partum CL of pregnancy is in measuring the post partum interval between parturition and commencement of the first mature cL. Hammond (1927) stated that CL of pregnancy in cattle remain at full size until 30 to 60 days post partum and prevent ovulation from occurring. Five of the GL accompanied by microscopic embryos were collected at 25, 27, 46, 58 and 70 days post partum. Corpora lutea of pregnancy at $<10$ days post partum are macroscopically similar to, although smaller than, cL with microscopic embryos, but are advancing in the histological regression that commences in late pregnancy. After 10 days post partum, they regress markedly. If the post partum GL of pregnancy were not differentiated histologically from the CL accompanied by microscopic embryos, the question would remain whether ovulation and new CL occur at $<10$ days post partum or GL of pregnancy remain at full-size until 25 to 70 days post partum.

\section{ACKNOWLEDGMENTS}

This article is derived from a doctoral dissertation submitted to Washington 
State University. The research was part of an investigation conducted by $\mathbf{H}$. K. Buechner who was supported by the National Science Foundation. I am grateful to Dr Buechner for the opportunity to participate in the study and use the ovary collection for my research, for his encouragement and guidance, and his help in preparing this article.

Mrs J. Buechner, W. Leuthold, R. Schloeth and S. Ruhweza contributed observations and helped collect specimens. The Uganda Game Department granted special permission to collect kob. H. W. Mossman and E. S. E. Hafez provided opportunities to do histological studies in their laboratories and gave me valuable advice.

\section{REFERENCES}

Armstrong, D. T. \& Hansel, W. (1959) Alteration of the bovine estrous cycle with oxytocin. 7. Dairy Sci. 43, 533.

BuECHNER, H. K. (1961a) Territorial behavior in Uganda kob. Science, N.Y. 133, 698.

Burghner, H. K. (1961b) Unilateral implantation in the Uganda kob. Nature, Lond. 190, 738.

Burchner, H. K. (1963) Territoriality as a behavioral adaptation to environment in Uganda kob. Proc. 16th Int. Congr. Zool. 3, 59.

Buechner, H. K., Morrison, J. A. \& Leurhold, W. (1966) Reproduction in Uganda kob with special reference to behavior. Symp. zool. Soc. Lond. 15, 69.

Corner, G. W. (1919) On the origin of the corpus luteum of the sow from both granulosa and theca interna. Am. F. Anat. 25, 117.

Corner, G. W. (1921) Cyclic changes in the ovaries and uterus of the sow, and their relation to the mechanism of implantation. Contr. Embryol. 13, 117.

Foley, R. \& GREenstern, J. P. (1958) Cytological changes in the bovine corpus luteum during early pregnancy. In: Reproduction and Infertility, pp. 88-96. Ed. F. Gassner. Pergamon Press, New York.

Hammond, J. (1927) The physiology of reproduction in the cow. Cambridge University Press.

Harrison, R. J. (1948) The changes occurring in the ovary of the goat during the estrous cycle and in early pregnancy. 7. Anat. 82, 21.

Marshall, F. H. A. (1903) The oestrous cycle and the formation of the corpus luteum in the sheep. Phil. Trans. R. Soc. B, 196, 47.

McNurr, G. (1924) The corpus luteum of the ox ovary in the relation to the oestrous cycle. 7 . Am. vet. med. Ass. 65, 556.

Morrison, J. A. \& BUechner, H. K. (1971) Reproductive phenomena during the post partum-preconception interval in the Uganda kob. F. Reprod. Fert. 26, 307.

Moss, S., Wrenn, T. R. \& Sykes, J. F. (1954) Some histological and histochemical observations on the bovine ovary during the estrous cycle. Anat. Rec. 120, 409.

Pimlotr, D. H. \& Mossman, H. W. (1959) A macroscopic ovary-sectioning method. F. Wildl. Mgmt, 23, 232.

Warbritton, V. (1934) The cytology of the corpora lutea of the ewe. F. Morph. 56, 181. 